\title{
The Broadcast Channel with Degraded Message Sets and Unreliable Conference
}

\author{
Dor Itzhak and Yossef Steinberg \\ Department of Electrical Engineering \\ Technion - Israel Institute of Technology \\ Haifa 32000, Israel \\ doritz@campus.technion.ac.il ysteinbe@ee.technion.ac.il
}

\begin{abstract}
As demonstrated in many recent studies, cooperation between users can greatly improve the performance of communication systems. Most of the works in the literature present models where all the users are aware of the resources available for cooperation. However, the scenario where cooperation links are sometimes unavailable or that some users cannot be updated whether the cooperation links are present or not, is more realistic in today's dynamic ad-hoc communication systems. In such a case we need coding schemes that exploit the cooperation links if they are present, and can still operate if cooperation is not possible. In this work we study the general broadcast channel model with degraded message sets and cooperation links that may be absent, and derive it's capacity region under such uncertainty conditions.
\end{abstract}

Index Terms-Broadcast channels, conferencing decoders, degraded message sets, unreliable cooperation

\section{INTRODUCTION}

Coding schemes that utilize cooperation links between users in a communication network can greatly improve the communication performance of the network. Unfortunately, in modern ad-hoc communication systems the availability of cooperation links is not guaranteed a priori. A typical scenario in such systems is that the users are aware of the possibility that some nodes in the network will serve as relays or helpers, but their help is unreliable and cannot be guaranteed a priori. Therefore, it is desired to derive coding schemes that exploit cooperation when it is available, but can still operate when they are not]

The broadcast channel is one of the main building blocks of multiuser communication networks, and as such draws much research efforts. The physically degraded broadcast channel with conferencing decoders was introduced and studied in [1], [2], and a related model with relay channel in [3], [4]. The non-degraded BC with degraded message sets and a conference link was presented and studied in [5]. Regarding the more realistic scenario, when the conference is unreliable, a physically degraded $\mathrm{BC}$ with a conference that may be absent was suggested and studied in [6], and later also in [7].

In this work we extend the results of [6], [7] to the general two user BC with degraded message sets and unreliable conference, building on [5]. Note that, while stochastically degraded BC is a well accepted model, that can be justified in some realistic scenarios (e.g, the scalar Gaussian BC), a physically degraded model is much harder to justify. Hence,

This work was supported by the Israel Science Foundation (grant No. 1285/16). with realistic cooperation problems in mind, the importance of the results presented here lie mainly in the extension of [6] and [7], to the more general channel model of [5], getting rid of the degradedness assumption. In Section II we define the model and in Section III we present the capacity region. Sketches of the proofs are given in Section IV.

\section{System Model AND Definitions}

Definition 1. An $\left(n, \mu_{0}, \mu_{0}^{\prime}, \mu_{1}, v_{1}, \epsilon\right)$ code for the DM-BC with degraded message sets and unreliable conference link consists of index sets $\mathcal{M}_{i}=\left\{1,2, \ldots, \mu_{i}\right\}, i=0,1, \mathcal{M}_{0}^{\prime}=$ $\left\{1,2, \ldots, \mu_{0}^{\prime}\right\}$ and $\mathcal{N}_{1}=\left\{1,2, \ldots, v_{1}\right\}$, an encoder mapping:

$$
f: \mathcal{M}_{0} \times \mathcal{M}_{0}^{\prime} \times \mathcal{M}_{1} \longrightarrow \mathcal{X}^{n}
$$

a conference mapping:

$$
\phi: \mathcal{Y}_{1}^{n} \longrightarrow \mathcal{N}_{1}
$$

and three decoder mappings

$$
\begin{aligned}
& g_{1}: \mathcal{Y}_{1}^{n} \longrightarrow \mathcal{M}_{0} \times \mathcal{M}_{0}^{\prime} \times \mathcal{M}_{1} \\
& g_{2}: \mathcal{Y}_{2}^{n} \longrightarrow \mathcal{M}_{0} \\
& g_{2}^{\prime}: \mathcal{Y}_{2}^{n} \times \mathcal{N}_{1} \longrightarrow \mathcal{M}_{0}^{\prime}
\end{aligned}
$$

such that the average probabilities of error if the conference link is present or not, denoted by $P_{e}^{\prime(n)}$ and $P_{e}^{(n)}$ respectively, do not exceed $\epsilon$. The common message $M_{0}$, the residual common message $M_{0}^{\prime}$ and the private message $M_{1}$, are uniformly distributed on the index set $\mathcal{M}_{0} \times \mathcal{M}_{0}^{\prime} \times \mathcal{M}_{1}$. The probabilities of error for the two cases are given by:

$$
\begin{aligned}
P_{e}^{\prime}(n) & =\frac{1}{\mu_{0} \mu_{0}^{\prime} \mu_{1}} \sum_{m_{0}, m_{0}^{\prime}, m_{1}} P_{Y_{1} Y_{2} \mid X}\left\{S^{\prime}{ }_{e} \mid f\left(m_{0}, m_{0}^{\prime}, m_{1}\right)\right\} \\
P_{e}^{(n)} & =\frac{1}{\mu_{0} \mu_{0}^{\prime} \mu_{1}} \sum_{m_{0}, m_{0}^{\prime}, m_{1}} P_{Y_{1} Y_{2} \mid X}\left\{S_{e} \mid f\left(m_{0}, m_{0}^{\prime}, m_{1}\right)\right\}
\end{aligned}
$$

where the sets $S_{e}, S_{e}^{\prime}$ are defined as

$$
\begin{aligned}
& S_{e} \triangleq\left\{\left(\boldsymbol{y}_{1}, \boldsymbol{y}_{2}\right): g_{1}\left(\boldsymbol{y}_{1}\right) \neq\left(m_{0}, m_{0}^{\prime}, m_{1}\right) \text { or } g_{2}\left(\boldsymbol{y}_{2}\right) \neq m_{0}\right\} \\
& S^{\prime}{ }_{e} \triangleq S_{e} \cup\left\{\left(\boldsymbol{y}_{1}, \boldsymbol{y}_{2}\right): g_{2}^{\prime}\left(\boldsymbol{y}_{2}, \phi\left(\boldsymbol{y}_{1}\right)\right) \neq m_{0}^{\prime}\right\}
\end{aligned}
$$

and for notational convenience, the dependence of $S_{e}$ and $S_{e}^{\prime}$ on the messages is dropped. The conference rate $C_{1}$ and the communication rates $\left(R_{0}, R_{0}^{\prime}, R_{1}\right)$ are defined as:

$$
C_{1}=\frac{\log \left(v_{1}\right)}{n} \quad R_{k}=\frac{\log \left(\mu_{k}\right)}{n}, k=0,1 \quad R_{0}^{\prime}=\frac{\log \left(\mu_{0}^{\prime}\right)}{n}
$$




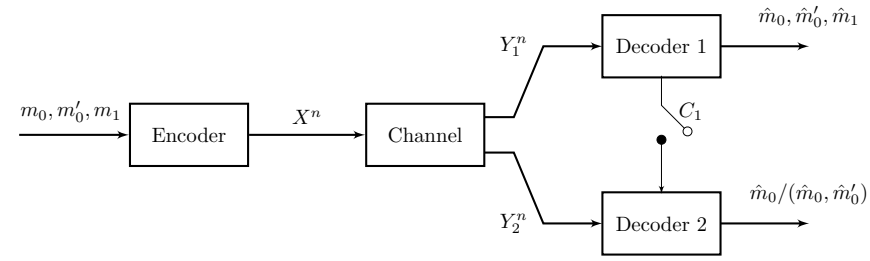

Figure 1. The broadcast channel with degraded message sets and unreliable conferencing decoders

A rate triple $\left(R_{0}, R_{0}^{\prime}, R_{1}\right)$ is said to be achievable, if for any $\epsilon>0, \gamma>0$ and sufficiently large $n$, there exists an $\left(n, 2^{n\left(R_{0}-\gamma\right)}, 2^{n\left(R_{0}^{\prime}-\gamma\right)}, 2^{n\left(R_{1}-\gamma\right)}, 2^{n\left(C_{1}+\gamma\right)}, \epsilon\right)$ code for the DM-BC with degraded message sets and unreliable conference link. The capacity region of the DM-BC with degraded message sets and unreliable conference link of capacity $C_{1}$ is the closure of the set of achievable rates $\left(R_{0}, R_{0}^{\prime}, R_{1}\right)$ for a given $C_{1}$, and is denoted by $\mathcal{C}$.

\section{CAPACITY REGION FOR THE BC WITH DEGRADED MESSAGE SETS AND UNRELIABLE CONFERENCE}

Let $\mathcal{R}^{i}$ be the set of all rate triples $\left(R_{0}, R_{0}^{\prime}, R_{1}\right)$ satisfying:

$$
\begin{aligned}
R_{0} & \leq I\left(U ; Y_{2}\right) \\
R_{0}^{\prime} & \leq I\left(V ; Y_{2} \mid U\right)+C_{1} \\
R_{1} & \leq I\left(X ; Y_{1} \mid U V\right) \\
R_{0}^{\prime}+R_{1} & \leq I\left(X ; Y_{1} \mid U\right) \\
R_{0}+R_{0}^{\prime}+R_{1} & \leq I\left(X ; Y_{1}\right)
\end{aligned}
$$

for some joint distribution $p(u, v, x) p\left(y_{1}, y_{2} \mid x\right)$. Consider some special cases of this region:

Case 1. BC with degraded message sets - In this model, there is no residual common message, i.e. $R_{0}^{\prime}=0$, and no intention to use the conference link. Here we choose $V=\emptyset$ to get the capacity region of the $\mathrm{BC}$ with degraded message sets first solved in [8]. Denote this region by $\mathcal{C}_{B C}$.

Case 2. BC with conferencing decoders and degraded message sets - In this model, there is no common message, i.e. $R_{0}=0$, so there is no intention to communicate if the conference link is absent. Here we choose $U=\emptyset$ to get the capacity region of the non-degraded $\mathrm{BC}$ with degraded message sets and conferencing decoders presented in [5]. Denote this region by $\mathcal{C}_{B C C}$ (BCC for $\mathrm{BC}$ with Conference).

Case 3. BC with unreliable conference link and common messages only - This special case not yet treated before and presented here for the sake of completeness. In this model, there is no private message, i.e. $R_{1}=0$, so there are only common messages to communicate. Here we choose $V=X$ :

$$
\begin{aligned}
& R_{0} \leq I\left(U ; Y_{2}\right) \\
& R_{0}^{\prime} \leq \min \left\{I\left(X ; Y_{1} \mid U\right), I\left(X ; Y_{2} \mid U\right)+C_{1}\right\} \\
R_{0}+ & R_{0}^{\prime} \leq I\left(X ; Y_{1}\right)
\end{aligned}
$$

for some joint distribution $p(u, x) p\left(y_{1}, y_{2} \mid x\right)$. Denote this region by $\mathcal{C}_{n o R_{1}}$. We claim that this is the capacity region for this channel with $R_{1}=0$. For detailed proof see Part D of Section IV.
Case 4. Degraded BC with conference link that may be absent - Assume that decoder 2 is physically degraded with respect to decoder 1 . In that case, the region coincides with the capacity region of the degraded $\mathrm{BC}$ with conference link that may be absent presented in [6], by treating the private messages as degraded message sets as decoder 1 can recover the private message intended to decoder 2 .

Let $\mathcal{R}^{o}$ be the set of all rate triples $\left(R_{0}, R_{0}^{\prime}, R_{1}\right)$ satisfying:

$$
\begin{aligned}
R_{0} & \leq I\left(U ; Y_{2}\right) \\
R_{0}+R_{0}^{\prime} & \leq I\left(U V ; Y_{2}\right)+C_{1} \\
R_{0}+R_{0}^{\prime}+R_{1} & \leq I\left(X ; Y_{1}\right) \\
R_{0}+R_{0}^{\prime}+R_{1} & \leq I\left(U ; Y_{2}\right)+I\left(X ; Y_{1} \mid U\right) \\
R_{0}+R_{0}^{\prime}+R_{1} & \leq I\left(U V ; Y_{2}\right)+C_{1}+I\left(X ; Y_{1} \mid U V\right) \\
R_{0}, R_{0}^{\prime}, R_{1} & \geq 0
\end{aligned}
$$

for some joint distribution $p(u, v, x) p\left(y_{1}, y_{2} \mid x\right)$.

Theorem 1. For the DM-BC with degraded message sets and unreliable conference link, the capacity region is given by

$$
\mathcal{C}=\mathcal{R}^{o}=\mathcal{R}^{i}
$$

Outline of the proof: The region $\mathcal{R}^{i}$ is an achievable region, i.e $\mathcal{R}^{i} \subseteq \mathcal{C}$; the proof of the direct part is quite similar to the proof in [6] - the encoder utilizes superposition coding and use binning for the residual message. A sketch of the converse part is given in Section IV. To complete the proof we will show the equivalence of those regions, i.e. $\mathcal{R}^{i}=\mathcal{R}^{o}$.

Example. Consider the AWGN BC with degraded message sets and unreliable link with capacity $C_{1}$ :

$$
Y_{i}=X+Z_{i} \quad Z_{i} \sim \mathcal{N}\left(0, N_{i}\right) \quad i=1,2
$$

where $N_{2}>N_{1}$, the noise signals $Z_{1}$ and $Z_{2}$ are independent and an input power constraint $E\left[X^{2}\right] \leq P$. Denote the classical AWGN capacity by:

$$
\mathcal{C}(x)=\frac{1}{2} \log (1+x)
$$

The capacity region for this model is given by the set of all rate triples $\left(R_{0}, R_{0}^{\prime}, R_{1}\right)$ satisfying:

$$
\begin{aligned}
R_{0} & \leq \mathcal{C}\left(\frac{\alpha_{0} P}{N_{2}+\left[\alpha_{0}^{\prime}+\alpha_{1}\right] P}\right) \\
R_{0}^{\prime} & \leq \mathcal{C}\left(\frac{\alpha_{0}^{\prime} P}{N_{2}+\alpha_{1} P}\right)+C_{1} \\
R_{1} & \leq \mathcal{C}\left(\frac{\alpha_{1} P}{N_{1}}\right) \\
R_{0}^{\prime}+R_{1} & \leq \mathcal{C}\left(\frac{\left[\alpha_{0}^{\prime}+\alpha_{1}\right] P}{N_{1}}\right)
\end{aligned}
$$

where $\alpha_{0}, \alpha_{0}^{\prime}, \alpha_{1} \geq 0$ and $\alpha_{0}+\alpha_{0}^{\prime}+\alpha_{1}=1$. For detailed proof see Part E of Section IV.

\section{PROOFS}

We will start with the proof of equivalence, i.e. $\mathcal{R}^{o}=\mathcal{R}^{i}$, then we give the converse and the direct part. In order to prove $\mathcal{R}^{o}=\mathcal{R}^{i}$ recall some definitions and lemmas from convex analysis. See [9] for the definition of extreme points, and [10] for the properties of polytopes.

Definitions: Let $S$ be a set of points in $\mathbb{R}^{k}$, i.e. $S \subseteq \mathbb{R}^{k}$. 
- A point $\mathrm{x} \in S$ is called an extreme point of $S$ if there do not exist $\mathbf{x}_{1}, \mathbf{x}_{2} \in S$ and $\lambda \in(0,1)$ where $\mathbf{x}_{1} \neq \mathbf{x}_{2}$, such that $\mathbf{x}=\lambda \mathbf{x}_{1}+(1-\lambda) \mathbf{x}_{2}$.

- Let $\operatorname{ext}(S)$ be the set of all extreme points of $S$.

- Let $\operatorname{conv}(S)$ be the convex hull of $S$.

- Let $\mathbf{A} \in \mathbb{R}^{m \times k}, \mathbf{b} \in \mathbb{R}^{m}$. Then $P \triangleq\left\{\mathbf{x} \in \mathbb{R}^{k} \mid \mathbf{A x} \leq \mathbf{b}\right\}$ is called a convex $\mathcal{H}$-generalized polytope ( $\mathcal{H}$ for halfspace). If in addition $P$ is bounded it called a convex $\mathcal{H}$-polytope.

- Let $\left\{\mathbf{x}_{1}, \ldots, \mathbf{x}_{l}\right\}$ be a set of points in $\mathbb{R}^{k}$. Then $P \triangleq$ $\operatorname{conv}\left(\left\{\mathbf{x}_{1}, \ldots, \mathbf{x}_{l}\right\}\right)$ is called a convex $\mathcal{V}$-polytope $(\mathcal{V}$ for vertex).

Lemma 2. Let $S \subseteq \mathbb{R}^{k}$ be a compact convex set, then

$$
S=\operatorname{conv}(\operatorname{ext}(S))
$$

Lemma 2 is common in convex analysis, see Corollary 18.5.1 on page 167 in [9].

Lemma 3. Main theorem of polytopes theory - Let $P \subseteq \mathbb{R}^{k}$ be a convex $\mathcal{H}$-polytope, then it has an equivalent representation as a convex $\mathcal{V}$-polytope by it's own vertices.

Lemma 3 can be found in [10], and it's proof can be found in [9] Theorem 19.1 and Corollary 19.1.1.

\section{A. Equivalence proof}

It is straight forward to prove that $\mathcal{R}^{i} \subseteq \mathcal{R}^{o}$ - for every $p(u, v, x)$, the inequalities satisfied in $\mathcal{R}^{i}$ imply that the inequalities in $\mathcal{R}^{o}$ are also satisfied. In order to prove $\mathcal{R}^{o} \subseteq \mathcal{R}^{i}$ we cannot use the last argument. Instead we will prove that $\operatorname{ext}\left(\mathcal{R}^{o}\right) \subseteq \mathcal{R}^{i}$ and then use Lemma 2 and the convexity property of those regions to conclude:

$$
\mathcal{R}^{o}=\operatorname{conv}\left(\operatorname{ext}\left(\mathcal{R}^{o}\right)\right) \subseteq \operatorname{conv}\left(\mathcal{R}^{i}\right)=\mathcal{R}^{i}
$$

Let $\left(R_{0}, R_{0}^{\prime}, R_{1}\right) \in \operatorname{ext}\left(\mathcal{R}^{o}\right)$. If $R_{0}=0$ for example, then in order to show that $\left(0, R_{0}^{\prime}, R_{1}\right) \in \mathcal{R}^{i}$ we will prove that $\mathcal{R}^{o}$ is tight on the intersection of $\mathcal{R}^{o}$ with the hyper-plane $R_{0}=0$, and similarly for $R_{0}^{\prime}=0$ and $R_{1}=0$. We expect to get $2 \mathrm{D}$ capacity regions, which correspond to the special cases presented in Section III after the definition of $\mathcal{R}^{i}$.

Lemma 4. Let

$$
\mathcal{R}^{o}\left(R_{0}=0\right) \triangleq\left\{\left(R_{0}^{\prime}, R_{1}\right) \mid\left(0, R_{0}^{\prime}, R_{1}\right) \in \mathcal{R}^{o}\right\}
$$

Define $\mathcal{R}^{o}\left(R_{0}^{\prime}=0\right)$ and $\mathcal{R}^{o}\left(R_{1}=0\right)$ similarly. Then

$$
\begin{aligned}
& \mathcal{R}^{o}\left(R_{0}=0\right)=\mathcal{C}_{B C C} \\
& \mathcal{R}^{o}\left(R_{0}^{\prime}=0\right)=\mathcal{C}_{B C} \\
& \mathcal{R}^{o}\left(R_{1}=0\right)=\mathcal{C}_{n o R_{1}}
\end{aligned}
$$

The proof of Lemma 4 is given at the end of this subsection. We proceed to prove that $\operatorname{ext}\left(\mathcal{R}^{o}\right) \subseteq \mathcal{R}^{i}$. Let $\left(R_{0}, R_{0}^{\prime}, R_{1}\right) \in$ $\operatorname{ext}\left(\mathcal{R}^{o}\right)$. If $R_{0}=0$, then from the fact that $\mathcal{C}_{B C}$ is a special case of $\mathcal{R}^{i}$, and from Lemma 4 above we can state

$$
\text { If }\left(0, R_{0}^{\prime}, R_{1}\right) \in \operatorname{ext}\left(\mathcal{R}^{o}\right) \Rightarrow\left(0, R_{0}^{\prime}, R_{1}\right) \in \mathcal{R}^{i}
$$

If $R_{0}^{\prime}=0$ or $R_{1}=0$ we can do the same. The remaining extreme points to be treated are in the positive orthant

$$
\mathbb{R}_{++}^{3} \triangleq\left\{\left(R_{0}, R_{0}^{\prime}, R_{1}\right) \in \mathbb{R}^{k} \mid R_{0}, R_{0}^{\prime}, R_{1}>0\right\}
$$

To find them all we will examine an arbitrary region in $\mathcal{R}^{o}$. Let $(U, V, X) \sim p(u, v, x)$ be any distribution. It defines a convex bounded $\mathcal{H}$-polytope - an intersection of eight halfspaces defined by the inequalities (2). By Lemma 3 , it has an equivalent representation as a $\mathcal{V}$-polytope of it's own vertices, and those vertices are the only candidates as extreme points of $\mathcal{R}^{o}$. Each vertex is obtained by only three (out of eight) linearly independent active inequalities, as three linearly independent equations define a specific point in $\mathbb{R}_{++}^{3}$. If there are less than three independent active inequalities, we can have a straight line (two active inequalities), a hyper-plane (one active inequality) or interior point (no active inequalities) - but not a vertex. We are interested only in the vertices in $\mathbb{R}_{++}^{3}$ if there exist one - so inequalities (2f) are in-active. Note that if there are no vertices in $\mathbb{R}_{++}^{3}$, then all the vertices has already been treated and proved to be in $\mathcal{R}^{i}$. The three inequalities 2c)-2e are all dependent, thus only one of them is active (unless there is a redundancy, but it does not affect the proof) thus inequalities (2a) and (2b) are surely active. Thus, for such a vertex, we have:

$$
\begin{aligned}
R_{0} & =I\left(U ; Y_{2}\right) \\
R_{0}+R_{0}^{\prime} & =I\left(U V ; Y_{2}\right)+C_{1} \\
R_{0}+R_{0}^{\prime}+R_{1} \leq I\left(X ; Y_{1}\right) & \leq\left(X ; Y_{1} \mid U\right) \\
R_{0}+R_{0}^{\prime}+R_{1} \leq I\left(U ; Y_{2}\right)+I(X) & \leq I\left(U V ; Y_{2}\right)+C_{1}+I\left(X ; Y_{1} \mid U V\right)
\end{aligned}
$$

where either (4c), (4d) or (4e) is active. Subtract (4b) from (4e), and subtract (4a) from (4b), (4d) to get an alternative representation of the same vertex:

$$
\begin{aligned}
R_{0} & =I\left(U ; Y_{2}\right) \\
R_{0}^{\prime} & =I\left(V ; Y_{2} \mid U\right)+C_{1} \\
R_{1} & \leq I\left(X ; Y_{1} \mid U V\right) \\
R_{0}^{\prime}+R_{1} & \leq I\left(X ; Y_{1} \mid U\right) \\
R_{0}+R_{0}^{\prime}+R_{1} & \leq I\left(X ; Y_{1}\right)
\end{aligned}
$$

where either (5c), (5d) or (5e) is active. This alternative representation of the vertex obeys (1), implying that this vertex is a point in the polytope induced from the same distribution $p(u, v, x)$ in $\mathcal{R}^{i}$. Thus for every polytope in $\mathcal{R}^{o}$, all vertices in $\mathbb{R}_{++}^{3}$ were proved to be in $\mathcal{R}^{i}$, implying that $\operatorname{ext}\left(\mathcal{R}^{o}\right) \subseteq \mathcal{R}^{i}$. This complete the equivalence proof of the regions $\mathcal{R}^{o}=\mathcal{R}^{i}$. Proof of Lemma 4. We will show the proof for $\mathcal{R}^{\circ}\left(R_{0}^{\prime}=0\right)=$ $\mathcal{C}_{B C}$. The others are treated the same and thus omitted. We have shown that $\mathcal{C}_{B C}$ is a special case of $\mathcal{R}^{i}$, and obviously $\mathcal{R}^{i} \subseteq \mathcal{R}^{o}$ - thus we have $\mathcal{C}_{B C} \subseteq \mathcal{R}^{o}\left(R_{0}^{\prime}=0\right)$. To show that $\mathcal{R}^{o}\left(R_{0}^{\prime}=0\right) \subseteq \mathcal{C}_{B C}$, consider $\mathcal{R}^{o}\left(R_{0}^{\prime}=0\right)$. It contains all rates $\left(R_{0}, R_{1}\right)$ satisfying:

$$
\begin{aligned}
R_{0} & \leq I\left(U ; Y_{2}\right) \\
R_{0}+R_{1} & \leq I\left(X ; Y_{1}\right) \\
R_{0}+R_{1} & \leq I\left(U ; Y_{2}\right)+I\left(X ; Y_{1} \mid U\right) \\
R_{0}+R_{1} & \leq I\left(U V ; Y_{2}\right)+C_{1}+I\left(X ; Y_{1} \mid U V\right)
\end{aligned}
$$

for some joint distribution $p(u, v, x) p\left(y_{1}, y_{2} \mid x\right)$. Let $(U, V, X) \sim p^{*}(u, v, x)$ be any distribution that defines a 2D-polytope denoted by $P_{1}$. Our goal is to prove this 
2D-polytope is also in $\mathcal{C}_{B C}$. Recall $\mathcal{C}_{B C}$ - it contains all rates $\left(R_{0}, R_{1}\right)$ satisfying:

$$
\begin{aligned}
R_{0} & \leq I\left(U ; Y_{2}\right) \\
R_{0}+R_{1} & \leq I\left(X ; Y_{1}\right) \\
R_{0}+R_{1} & \leq I\left(U ; Y_{2}\right)+I\left(X ; Y_{1} \mid U\right)
\end{aligned}
$$

for some joint distribution $p(u, x) p\left(y_{1}, y_{2} \mid x\right)$.

Consider the 2D-polytope $P_{2}$ contained in $\mathcal{C}_{B C}$, defined by $(U, X) \sim p^{*}(u, x)$. We have that $P_{1} \subseteq P_{2}$ as both of them obey the same three inequalities $(6 \mathrm{ab})-(\sqrt{6 \mathrm{c}})$, and that $P_{1}$ has one more inequality (6d) to hold. It is true to any $P_{1}$ in $\mathcal{R}^{o}\left(R_{0}^{\prime}=0\right)$, thus $\mathcal{R}^{o}\left(R_{0}^{\prime}=0\right) \subseteq \mathcal{C}_{B C}$.

\section{B. Converse Part}

Let $\left(2^{n R_{0}}, 2^{n R_{0}^{\prime}}, 2^{n R_{1}}, n\right)$ be any sequence of codes for the DM-BC with degraded message sets and unreliable conference link that satisfies

$$
\lim _{n \rightarrow \infty} P_{e}^{(n)}=0 \quad \lim _{n \rightarrow \infty} P_{e}^{\prime(n)}=0
$$

We have to show that the inequalities (2) hold, for some pmf $p(u, v, x)$. Applying the Fano's inequality:

$$
\begin{aligned}
& n\left(R_{0}-\epsilon_{2, n}\right) \leq I\left(M_{0} ; Y_{2}^{n}\right) \\
& =\sum_{i=1}^{n} I\left(M_{0} ; Y_{2, i} \mid Y_{2, i+1}^{n}\right) \\
& \leq \sum_{i=1}^{n} I\left(M_{0} Y_{2, i+1}^{n} Y_{1}^{i-1} ; Y_{2, i}\right) \\
& n\left(R_{0}+R_{0}^{\prime}-\epsilon_{2, n}^{\prime}\right) \leq I\left(M_{0} M_{0}^{\prime} ; Y_{2}^{n}, \phi\left(Y_{1}^{n}\right)\right) \\
& =I\left(M_{0} M_{0}^{\prime} ; Y_{2}^{n}\right)+I\left(M_{0} M_{0}^{\prime} ; \phi\left(Y_{1}^{n}\right) \mid Y_{2}^{n}\right) \\
& \leq \sum_{i=1}^{n} I\left(M_{0} M_{0}^{\prime} ; Y_{2, i} \mid Y_{2, i+1}^{n}\right)+H\left(\phi\left(Y_{1}^{n}\right)\right) \\
& \leq \sum_{i=1}^{n} I\left(M_{0} M_{0}^{\prime} Y_{2, i+1}^{n} ; Y_{2, i}\right)+n C_{1} \\
& \leq \sum_{i=1}^{n} I\left(M_{0} M_{0}^{\prime} Y_{2, i+1}^{n} Y_{1}^{i-1} ; Y_{2, i}\right)+n C_{1} \\
& n\left(R_{0}+R_{0}^{\prime}+R_{1}-\epsilon_{1, n}\right) \leq I\left(M_{0} M_{0}^{\prime} M_{1} ; Y_{1}^{n}\right) \\
& =\sum_{i=1}^{n} I\left(M_{0} M_{0}^{\prime} M_{1} ; Y_{1, i} \mid Y_{1}^{i-1}\right) \\
& \leq \sum_{i=1}^{n} I\left(X_{i} M_{0} M_{0}^{\prime} M_{1} Y_{1}^{i-1} ; Y_{1, i}\right) \\
& =\sum_{i=1}^{n} I\left(X_{i} ; Y_{1, i}\right)
\end{aligned}
$$

where $\epsilon_{1, n}, \epsilon_{2, n}, \epsilon^{\prime}{ }_{2, n}$ tend to zero as $n \rightarrow \infty$. To prove inequalities (2d) and (2e) we bound:

$$
\begin{aligned}
& n\left(R_{0}+R_{0}^{\prime}+R_{1}-\epsilon_{2, n}^{\prime}-\epsilon_{1, n}\right) \\
& \leq I\left(M_{0} ; Y_{2}^{n}\right)+I\left(M_{0}^{\prime} M_{1} ; Y_{1}^{n} \mid M_{0}\right) \\
& =\sum_{i=1}^{n} I\left(M_{0} ; Y_{2, i} \mid Y_{2, i+1}\right)+\sum_{i=1}^{n} I\left(M_{0}^{\prime} M_{1} ; Y_{1, i} \mid M_{0} Y_{1}^{i-1}\right) \\
& \leq \sum_{i=1}^{n} I\left(M_{0} Y_{2, i+1}^{n} ; Y_{2, i}\right) \\
& +\sum_{i=1}^{n} I\left(M_{0}^{\prime} M_{1} Y_{2, i+1}^{n} ; Y_{1, i} \mid M_{0} Y_{1}^{i-1}\right) \\
& =\sum_{i=1}^{n} I\left(M_{0} Y_{2, i+1}^{n} ; Y_{2, i}\right)+\sum_{i=1}^{n} I\left(Y_{2, i+1}^{n} ; Y_{1, i} \mid M_{0} Y_{1}^{i-1}\right) \\
& +\sum_{i=1}^{n} I\left(M_{0}^{\prime} M_{1} ; Y_{1, i} \mid M_{0} Y_{1}^{i-1} Y_{2, i+1}^{n}\right) \\
& \stackrel{(a)}{=} \sum_{i=1}^{n} I\left(M_{0} Y_{2, i+1} ; Y_{2, i}\right)+\sum_{i=1}^{n} I\left(Y_{1}^{i-1} ; Y_{2, i} \mid M_{0} Y_{2, i+1}{ }^{n}\right) \\
& +\sum_{i=1}^{n} I\left(X_{i} M_{0}^{\prime} M_{1} ; Y_{1, i} \mid M_{0} Y_{1}^{i-1} Y_{2, i+1}^{n}\right) \\
& =\sum_{i=1}^{n} I\left(M_{0} Y_{2, i+1}^{n} Y_{1}^{i-1} ; Y_{2, i}\right) \\
& +\sum_{i=1}^{n} I\left(X_{i} ; Y_{1, i} \mid M_{0} Y_{1}^{i-1} Y_{2, i+1}^{n}\right) \\
& n\left(R_{0}+R_{0}^{\prime}+R_{1}-\epsilon_{2, n}^{\prime}-\epsilon_{1, n}\right) \\
& \leq I\left(M_{0} M_{0}^{\prime} ; Y_{2}^{n}, \phi\left(Y_{1}^{n}\right)\right)+I\left(M_{1} ; Y_{1}^{n} \mid M_{0} M_{0}^{\prime}\right) \\
& \stackrel{(b)}{\leq} \sum_{i=1}^{n} I\left(M_{0} M_{0}^{\prime} Y_{2, i+1}^{n} ; Y_{2, i}\right)+n C_{1} \\
& +\sum_{i=1}^{n} I\left(M_{1} Y_{2, i+1}^{n} ; Y_{1, i} \mid M_{0} M_{0}^{\prime} Y_{1}^{i-1}\right) \\
& \leq \sum_{i=1}^{n} I\left(M_{0} M_{0}^{\prime} Y_{2, i+1}^{n} ; Y_{2, i}\right)+n C_{1} \\
& +\sum_{i=1}^{n} I\left(Y_{2, i+1}^{n} ; Y_{1, i} \mid M_{0} M_{0}^{\prime} Y_{1}^{i-1}\right) \\
& +\sum_{i=1}^{n} I\left(M_{1} ; Y_{1, i} \mid M_{0} M_{0}^{\prime} Y_{1}^{i-1} Y_{2, i+1}^{n}\right) \\
& \stackrel{(a)}{=} \sum_{i=1}^{n} I\left(M_{0} M_{0}^{\prime} Y_{2, i+1}^{n} ; Y_{2, i}\right)+n C_{1} \\
& +\sum_{i=1}^{n} I\left(Y_{1}^{i-1} ; Y_{2, i} \mid M_{0} M_{0}^{\prime} Y_{2, i+1}^{n}\right) \\
& +\sum_{i=1}^{n} I\left(X_{i} M_{1} ; Y_{1, i} \mid M_{0} M_{0}^{\prime} Y_{1}^{i-1} Y_{2, i+1}^{n}\right) \\
& =\sum_{i=1}^{n} I\left(M_{0} Y_{2, i+1}^{n} Y_{1}^{i-1} M_{0}^{\prime} ; Y_{2, i}\right)+n C_{1} \\
& +\sum_{i=1}^{n} I\left(X_{i} ; Y_{1, i} \mid M_{0} Y_{1}^{i-1} Y_{2, i+1}^{n} M_{0}^{\prime}\right)
\end{aligned}
$$


where $(a)$ is due to Csiszar sum identity and the fact that $X_{i}$ is a deterministic function of the messages, and $(b)$ is from inequality (8) above. Finally back to inequalities (7)-(11) and define $U_{i}=\left(M_{0} Y_{2, i+1}^{n} Y_{1}^{i-1}\right), V_{i}=M_{0}^{\prime}$. Then define also a time-sharing random variable uniformly distributed $Q \sim$ Uniform $[1: n]$ independent of all R.V, and taking the limit $n \rightarrow \infty$ to complete the converse proof.

\section{Direct Part}

The encoder utilizes a superposition coding scheme and uses binning for the residual message.

Codebook Generation: Fix $P_{U} P_{V \mid U} P_{X \mid U V}$. Generate the codebook $\mathcal{C}$ as follows:

1) Generate $2^{n R_{0}}$ independent codewords

$$
\boldsymbol{u}\left(m_{0}\right) \sim \prod_{i=1}^{n} P_{U}\left(u_{i}\right)
$$

where $m_{0} \in\left[1: 2^{n R_{0}}\right]$.

2) For each codeword $\boldsymbol{u}\left(m_{0}\right)$, generate $2^{n R_{0}^{\prime}}$ conditionally independent codewords

$$
\boldsymbol{v}\left(m_{0}, m_{0}^{\prime}\right) \sim \prod_{i=1}^{n} P_{V \mid U}\left(v \mid u_{i}\left(m_{0}\right)\right)
$$

where $m_{0}^{\prime} \in\left[1: 2^{n R_{0}^{\prime}}\right]$.

3) For each couple of codewords $\boldsymbol{u}\left(m_{0}\right), \boldsymbol{v}\left(m_{0}, m_{0}^{\prime}\right)$, generate $2^{n R_{1}}$ conditionally independent codewords

$$
\boldsymbol{x}\left(m_{0}, m_{0}^{\prime}, m_{1}\right) \sim \prod_{i=1}^{n} P_{X \mid U V}\left(x_{i} \mid u_{i}\left(m_{0}\right), v_{i}\left(m_{0}, m_{0}^{\prime}\right)\right)
$$

where $m_{1} \in\left[1: 2^{n R_{1}}\right]$.

4) Divide the residual message set $\left[1: 2^{n R_{0}^{\prime}}\right]$ into $2^{n C_{1}}$ equal bins, each bin contains $2^{n\left(R_{0}^{\prime}-C_{1}\right)}$ messages. Denote by $\operatorname{bin}(m)$ the bin index of any message $m \in$ $\left[1: 2^{n R_{0}^{\prime}}\right]$.

This defines the codebook:

$\mathcal{C}=\left\{\begin{array}{c}{\left[\boldsymbol{u}\left(m_{0}\right), \boldsymbol{v}\left(m_{0}, m_{0}^{\prime}\right), \boldsymbol{x}\left(m_{0}, m_{0}^{\prime}, m_{1}\right)\right]} \\ \left(m_{0}, m_{0}^{\prime}, m_{1}\right) \in\left[1: 2^{n R_{0}}\right] \times\left[1: 2^{n R_{0}^{\prime}}\right] \times\left[1: 2^{n R_{1}}\right]\end{array}\right\}$

Encoding and decoding scheme: Encoder: Let $\left(m_{0}, m_{0}^{\prime}, m_{1}\right) \in\left[1: 2^{n R_{0}}\right] \times\left[1: 2^{n R_{0}^{\prime}}\right] \times\left[1: 2^{n R_{1}}\right]$ be the messages to be sent. The encoder transmits $\boldsymbol{x}\left(m_{0}, m_{0}^{\prime}, m_{1}\right)$ from codebook $\mathcal{C}$.

Decoder 1 finds the unique triplet $\left(\hat{m}_{0}, \hat{m}_{0}^{\prime}, \hat{m}_{1}\right)$ such that

$$
\left(\boldsymbol{u}\left(\hat{m}_{0}\right), \boldsymbol{v}\left(\hat{m}_{0}, \hat{m}_{0}^{\prime}\right), \boldsymbol{x}\left(\hat{m}_{0}, \hat{m}_{0}^{\prime}, \hat{m}_{1}\right), \boldsymbol{y}_{1}\right) \in \mathcal{T}_{e}^{(n)}
$$

By standard techniques, decoder 1 decodes $\left(m_{0}, m_{0}^{\prime}, m_{1}\right)$ correctly, with an arbitrarily small probability of error, if

$$
\begin{aligned}
R_{1} & \leq I\left(X ; Y_{1} \mid U V\right) \\
R_{0}^{\prime}+R_{1} & \leq I\left(X ; Y_{1} \mid U\right) \\
R_{0}+R_{0}^{\prime}+R_{1} & \leq I\left(X ; Y_{1}\right)
\end{aligned}
$$

In the end of the transmission and if the conference link is present, decoder 1 sends to decoder 2 the estimated residual message's bin index $\operatorname{bin}\left(\hat{m}_{0}^{\prime}\right)$.

Decoder 2 - the operation of decoder 2 depends on whether the conference link is present

If the conference link is absent, decoder 2 finds the unique message $\check{m}_{0}$ such that

$$
\left(\boldsymbol{u}\left(\check{m}_{0}\right), \boldsymbol{y}_{2}\right) \in \mathcal{T}_{e}^{(n)}
$$

By standard techniques, decoder 2 decodes $m_{0}$ correctly, with an arbitrarily small probability of error, if

$$
R_{0} \leq I\left(U ; Y_{2}\right)
$$

If the conference link is present, decoder 2 finds the unique couple of messages $\left(\check{m}_{0}, \check{m}_{0}^{\prime}\right)$ such that

$$
\left(\boldsymbol{u}\left(\check{m}_{0}\right), \boldsymbol{v}\left(\check{m}_{0}, \check{m}_{0}^{\prime}\right), \boldsymbol{y}_{2}\right) \in \mathcal{T}_{e}^{(n)}
$$

and where $\operatorname{bin}\left(\check{m}_{0}^{\prime}\right)=\phi\left(\boldsymbol{y}_{1}\right)$.

By standard techniques, decoder 2 decodes $\left(m_{0}, m_{0}^{\prime}\right)$ correctly, with an arbitrarily small probability of error, if

$$
\begin{aligned}
R_{0}^{\prime}-C_{1} & \leq I\left(V ; Y_{2} \mid U\right) \\
R_{0}+R_{0}^{\prime}-C_{1} & \leq I\left(U V ; Y_{2}\right)
\end{aligned}
$$

Note that inequality $13 \mathrm{c}$ is already satisfied, as inequalities (13a) and 13b are satisfied. The direct part follows by (12), (13a) and (13b).

Error Probability Analysis: Assume without loss of generality, that $\left(m_{0}, m_{0}^{\prime}, m_{1}\right)=(1,1,1)$ were sent, and that $\operatorname{bin}\left(m_{0}^{\prime}\right)=1$. Define the error event $\mathcal{E}_{1}$ :

$$
\mathcal{E}_{1}=\left\{\left(\hat{M}_{0}, \hat{M}_{0}^{\prime}, \hat{M}_{1}\right) \neq(1,1,1)\right\}
$$

Decoder 1 makes an error only if one or more of the following events occur:

$$
\begin{aligned}
& \mathcal{E}_{10}=\left\{\left(\boldsymbol{u}(1), \boldsymbol{v}(1,1), \boldsymbol{x}(1,1,1), \boldsymbol{y}_{1}\right) \notin \mathcal{T}_{e}^{(n)}\right\} \\
& \mathcal{E}_{11}=\bigcup_{\left(m_{0}, m_{0}^{\prime}, m_{1}\right) \neq(1,1,1)} \mathcal{E}\left(m_{0}, m_{0}^{\prime}, m_{1}\right)
\end{aligned}
$$

$\mathcal{E}\left(m_{0}, m_{0}^{\prime}, m_{1}\right)=\left\{\left(\boldsymbol{u}\left(m_{0}\right), \boldsymbol{v}\left(m_{0}, m_{0}^{\prime}\right), \boldsymbol{x}\left(m_{0}, m_{0}^{\prime}, m_{1}\right), \boldsymbol{y}_{1}\right) \in \mathcal{T}_{e}^{(n)}\right\}$ where the error events $\mathcal{E}\left(m_{0}, m_{0}^{\prime}, m_{1}\right)$ are divided into eight different groups, each group contains exponentially many error events of the same kind. The probability of $\mathcal{E}_{10}$ tends to zero as $n \rightarrow \infty$ by LLN, and the probability of the other error events tend to zero by the Packing Lemma [11] if:

$$
\begin{aligned}
R_{1} & \leq I\left(X ; Y_{1} \mid U V\right) \\
R_{0}^{\prime} & \leq I\left(X ; Y_{1} \mid U\right) \\
R_{0}^{\prime}+R_{1} & \leq I\left(X ; Y_{1} \mid U\right) \\
R_{0} & \leq I\left(X ; Y_{1}\right) \\
R_{0}+R_{1} & \leq I\left(X ; Y_{1}\right) \\
R_{0}+R_{0}^{\prime} & \leq I\left(X ; Y_{1}\right) \\
R_{0}+R_{0}^{\prime}+R_{1} & \leq I\left(X ; Y_{1}\right)
\end{aligned}
$$

Note that $14 \mathrm{~b}$ ) and $(14 \mathrm{~d}-14 \mathrm{f}$ are redundant as $114 \mathrm{c}$ and (14g) hold, respectively. The joint pmf of each group and the relevant rates constraint has to be satisfied in order to have $P(\mathcal{E}) \rightarrow 0$, is depicted in Table (I). The probability of error 


\begin{tabular}{|c|c|c|c|c|c|}
\hline $\mathcal{E}$ & $m_{0}$ & $m_{0}^{\prime}$ & $m_{1}$ & $p\left(\boldsymbol{u}, \boldsymbol{v}, \boldsymbol{x}, \boldsymbol{y}_{1}\right)$ & $P(\mathcal{E}) \rightarrow 0$ \\
\hline \hline $\mathcal{E}_{10}$ & 1 & 1 & 1 & $p(\boldsymbol{u}, \boldsymbol{v}, \boldsymbol{x}) p\left(\boldsymbol{y}_{1} \mid \boldsymbol{x}\right)$ & - \\
\hline $\mathcal{E}_{(1,1, *)}$ & 1 & 1 & $*$ & $p(\boldsymbol{u}, \boldsymbol{v}, \boldsymbol{x}) p\left(\boldsymbol{y}_{1} \mid \boldsymbol{u}, \boldsymbol{v}\right)$ & $\overline{14 \mathrm{a}}$ \\
\hline $\mathcal{E}_{(1, *, 1)}$ & 1 & $*$ & 1 & $p(\boldsymbol{u}, \boldsymbol{v}, \boldsymbol{x}) p\left(\boldsymbol{y}_{1} \mid \boldsymbol{u}\right)$ & $\overline{14 \mathrm{~b}}$ \\
\hline $\mathcal{E}(1, *, *)$ & 1 & $*$ & $*$ & $p(\boldsymbol{u}, \boldsymbol{v}, \boldsymbol{x}) p\left(\boldsymbol{y}_{1} \mid \boldsymbol{u}\right)$ & $\overline{14 \mathrm{c}}$ \\
\hline $\mathcal{E}(*, 1,1)$ & $*$ & 1 & 1 & $p(\boldsymbol{u}, \boldsymbol{v}, \boldsymbol{x}) p\left(\boldsymbol{y}_{1}\right)$ & $\overline{14 \mathrm{~d}}$ \\
\hline $\mathcal{E}(*, 1, *)$ & $*$ & 1 & $*$ & $p(\boldsymbol{u}, \boldsymbol{v}, \boldsymbol{x}) p\left(\boldsymbol{y}_{1}\right)$ & $\overline{14 \mathrm{e}}$ \\
\hline $\mathcal{E}(*, *, 1)$ & $*$ & $*$ & 1 & $p(\boldsymbol{u}, \boldsymbol{v}, \boldsymbol{x}) p\left(\boldsymbol{y}_{1}\right)$ & $\overline{14 \mathrm{f}}$ \\
\hline $\mathcal{E}(*, *, *)$ & $*$ & $*$ & $*$ & $p(\boldsymbol{u}, \boldsymbol{v}, \boldsymbol{x}) p\left(\boldsymbol{y}_{1}\right)$ & $\overline{14 \mathrm{~g}}$ \\
\hline
\end{tabular}

Table I

THE ERROR EVENTS OF DECODER 1

event $\mathcal{E}_{1}$ is upper bounded as:

$$
\begin{aligned}
P\left(\mathcal{E}_{1}\right) & \triangleq P\left\{\left(\hat{M}_{0}, \hat{M}_{0}^{\prime}, \hat{M}_{1}\right) \neq(1,1,1)\right\} \\
& =P\left(\mathcal{E}_{10} \cup \mathcal{E}_{11}\right) \leq P\left(\mathcal{E}_{10}\right)+P\left(\mathcal{E}_{11}\right)
\end{aligned}
$$

the first term tends to zero as $n \rightarrow \infty$ by LLN, and the second term tends to zero as $n \rightarrow \infty$ if (14a), (14c) and (14g) hold. Define the error event $\mathcal{E}_{2}$ :

$$
\mathcal{E}_{2}=\left\{\check{M}_{0} \neq 1\right\}
$$

If the conference link is absent, decoder 2 makes an error only if one or more of the following events occur:

$$
\begin{aligned}
& \mathcal{E}_{20}=\left\{\left(\boldsymbol{u}(1), \boldsymbol{y}_{2}\right) \notin \mathcal{T}_{e}^{(n)}\right\} \\
& \mathcal{E}_{21}=\left\{\left(\boldsymbol{u}\left(m_{0}\right), \boldsymbol{y}_{2}\right) \in \mathcal{T}_{e}^{(n)} \text { s.t. } m_{0} \neq 1\right\}
\end{aligned}
$$

Following the same analysis, the probability of error event $\mathcal{E}_{2}$ is upper bounded as:

$$
\begin{aligned}
P\left(\mathcal{E}_{2}\right) & \triangleq P\left\{\check{M}_{0} \neq 1\right\} \\
& =P\left(\mathcal{E}_{20} \cup \mathcal{E}_{21}\right) \leq P\left(\mathcal{E}_{20}\right)+P\left(\mathcal{E}_{21}\right)
\end{aligned}
$$

the first term tends to zero as $n \rightarrow \infty$ by LLN, and by Packing Lemma the second term tends to zero as $n \rightarrow \infty$ if

$$
R_{0} \leq I\left(U ; Y_{2}\right)
$$

Define the error event $\mathcal{E}_{2}^{\prime}$ :

$$
\mathcal{E}_{2}^{\prime}=\left\{\left(\check{M}_{0}, \check{M}_{0}^{\prime}\right) \neq(1,1)\right\}
$$

If the conference link is present, decoder 2 makes an error only if one or more of the following events occur:

$$
\begin{aligned}
\mathcal{E}^{\prime}{ }_{20}= & \left\{\left(\boldsymbol{u}(1), \boldsymbol{v}(1,1), \boldsymbol{y}_{2}\right) \notin \mathcal{T}_{e}^{(n)}\right\} \\
\mathcal{E}^{\prime}{ }_{21}= & \left\{\left(\boldsymbol{u}\left(m_{0}\right), \boldsymbol{v}\left(m_{0}, m_{0}^{\prime}\right), \boldsymbol{y}_{2}\right) \in \mathcal{T}_{e}^{(n)}\right. \\
& \text { s.t. } \left.\left(m_{0}, m_{0}^{\prime}\right) \neq(1,1), \operatorname{bin}\left(m_{0}^{\prime}\right)=1\right\}
\end{aligned}
$$

The probability of error event $\mathcal{E}^{\prime}{ }_{2} \cap \mathcal{E}_{1}^{C}$ is upper bounded as:

$$
\begin{aligned}
P\left(\mathcal{E}_{2}^{\prime} \cap \mathcal{E}_{1}^{C}\right) & =P\left(\left\{\left(\check{M}_{0}, \check{M}_{0}^{\prime}\right) \neq(1,1)\right\} \cap \mathcal{E}_{1}^{C}\right) \\
& =P\left(\left(\mathcal{E}^{\prime}{ }_{20} \cup \mathcal{E}^{\prime}{ }_{21}\right) \cap \mathcal{E}_{1}^{C}\right) \\
& \leq P\left(\mathcal{E}^{\prime}{ }_{20}\right)+P\left(\mathcal{E}^{\prime}{ }_{21} \cap \mathcal{E}_{1}^{C}\right)
\end{aligned}
$$

the first term tends to zero as $n \rightarrow \infty$ by LLN, and by Packing Lemma the second term tends to zero as $n \rightarrow \infty$ if

$$
\begin{aligned}
R_{0}^{\prime}-C_{1} & \leq I\left(V ; Y_{2} \mid U\right) \\
R_{0}+R_{0}^{\prime}-C_{1} & \leq I\left(U V ; Y_{2}\right)
\end{aligned}
$$

Note that $16 \mathrm{~b}$ is redundant as $(16 \mathrm{a}$ and $(15)$ hold. Finally $P_{e}^{(n)}$ and $P_{e}^{(n)}$ are upper bounded as:

$$
\begin{aligned}
P_{e}^{(n)} & =P\left(\mathcal{E}_{1} \cup \mathcal{E}_{2}\right) \leq P\left(\mathcal{E}_{1}\right)+P\left(\mathcal{E}_{2}\right) \\
P_{e}^{\prime(n)} & =P\left(\mathcal{E}_{1} \cup \mathcal{E}^{\prime}{ }_{2}\right)=P\left(\mathcal{E}_{1}\right)+P\left(\mathcal{E}^{\prime}{ }_{2} \cap \mathcal{E}_{1}^{C}\right)
\end{aligned}
$$

where both $P_{e}^{(n)}$ and $P_{e}^{\prime(n)}$ tend to zero if (2) hold. This complete the direct part.

\section{Proof for Case 3}

Let $\mathcal{R}_{n o R_{1}}^{i}$ be the set of all rate pairs $\left(R_{0}, R_{0}^{\prime}\right)$ satisfying:

$$
\begin{aligned}
R_{0} & \leq I\left(U ; Y_{2}\right) \\
R_{0}^{\prime} & \leq \min \left\{I\left(X ; Y_{1} \mid U\right), I\left(X ; Y_{2} \mid U\right)+C_{1}\right\} \\
R_{0}+R_{0}^{\prime} & \leq I\left(X ; Y_{1}\right)
\end{aligned}
$$

for some joint distribution $p(u, x) p\left(y_{1}, y_{2} \mid x\right)$. It is the same achievable region defined in Section III Case 3. Until proven otherwise, we shall treat this region only as an achievable region. Let $\mathcal{R}_{n o R_{1}}^{o}$ be the set of all rate pairs $\left(R_{0}, R_{0}^{\prime}\right)$ satisfying:

$$
\begin{aligned}
R_{0} & \leq I\left(U ; Y_{2}\right) \\
R_{0}+R_{0}^{\prime} & \leq I\left(U ; Y_{2}\right)+I\left(X ; Y_{1} \mid U\right) \\
R_{0}+R_{0}^{\prime} & \leq I\left(X ; Y_{2}\right)+C_{1} \\
R_{0}+R_{0}^{\prime} & \leq I\left(X ; Y_{1}\right)
\end{aligned}
$$

for some joint distribution $p(u, x) p\left(y_{1}, y_{2} \mid x\right)$.

Theorem 5. The capacity region for the DM-BC with unreliable conference link and common messages only is given by

$$
\mathcal{C}_{n o R_{1}}=\mathcal{R}_{n o R_{1}}^{o}=\mathcal{R}_{n o R_{1}}^{i}
$$

The proof of the capacity region for this $2 \mathrm{D}$ model stimulated the proof for the general model defined in Section II. The region $\mathcal{R}_{n o R_{1}}^{i}$ is achievable as a special case of $\mathcal{R}^{i}$, see Section III Case 3 . We proceed by proving that $\mathcal{R}_{n o R_{1}}^{o}=\mathcal{R}_{n o R_{1}}^{i}$ and then prove the converse part, i.e. $\mathcal{C}_{n o R_{1}} \subseteq \mathcal{R}_{n o R_{1}}^{o}$, which concludes that $\mathcal{C}_{n o R_{1}}=\mathcal{R}_{n o R_{1}}^{o}=\mathcal{R}_{n o R_{1}}^{i}$. It is straight forward to prove that $\mathcal{R}_{n o R_{1}}^{i} \subseteq \mathcal{R}_{n o R_{1}}^{o}$ - for every $p(u, x)$, the inequalities satisfied in $\mathcal{R}_{n o R_{1}}^{i}$ imply that the inequalities in $\mathcal{R}_{n o R_{1}}^{o}$ are also satisfied. In order to prove $\mathcal{R}_{n o R_{1}}^{o} \subseteq \mathcal{R}_{n o R_{1}}^{i}$ we cannot use the last argument. Instead we will examine the corner points of an arbitrary region in $\mathcal{R}_{n o R_{1}}^{o}$. Define the capacities:

$$
\begin{aligned}
& C_{0} \triangleq \max _{p(x)} \min \left\{I\left(X ; Y_{1}\right),\left(X ; Y_{2}\right)\right\} \\
& C_{0}^{\prime} \triangleq \max _{p(x)} \min \left\{I\left(X ; Y_{1}\right),\left(X ; Y_{2}\right)+C_{1}\right\}
\end{aligned}
$$

The line segments from the origin to $\left(C_{0}, 0\right)$ and $\left(0, C_{0}^{\prime}\right)$ are in $\mathcal{R}_{n o R_{1}}^{o}$ and $\mathcal{R}_{n o R_{1}}^{i}$. The remaining points to examine are $\left\{\left(R_{0}, R_{0}^{\prime}\right) \in \mathcal{R}_{n o R_{1}}^{o}: R_{0}, R_{0}^{\prime}>0\right\}$. Observe that for every $p(u, x)$, inequalities 17 define a triangle or a trapezoid. The only points of interest are the corner points like the point $\mathrm{A}$ in Fig. 2, as if we prove that a corner point is in $\mathcal{R}_{n o R_{1}}^{i}$, we can say that all the other points in the trapezoid are also in $\mathcal{R}_{n o R_{1}}^{i}$ using the convexity property. Note that in the triangle shape, there are no points of interest because the triangle is 


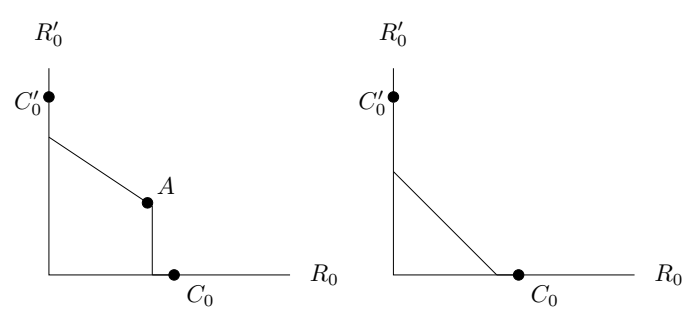

Figure 2. The optional region shapes in $\mathcal{R}_{n o R_{1}}^{o}$

surely contained in $\mathcal{R}_{n o R_{1}}^{i}$, as the triangle's hypotenuse edges intersect the axes on the line segments contained in $\mathcal{R}_{\text {no } R_{1}}^{i}$ and due to the convexity property. Each corner point $\mathrm{A}$ is the intersection of two linearly independent active constraints:

$$
\begin{aligned}
R_{0} & =I\left(U ; Y_{2}\right) \\
R_{0}+R_{0}^{\prime} & \leq I\left(U ; Y_{2}\right)+I\left(X ; Y_{1} \mid U\right) \\
R_{0}+R_{0}^{\prime} & \leq I\left(X ; Y_{2}\right)+C_{1} \\
R_{0}+R_{0}^{\prime} & \leq I\left(X ; Y_{1}\right)
\end{aligned}
$$

where $(18 \mathrm{a})$ and one of the three $(18 \mathrm{~b})-(18 \mathrm{~d})$ are active. Subtract (18a) from (18b and $118 \mathrm{c})$ to have that $\mathrm{A}$ is also $\mathcal{R}_{n o R_{1}}^{o}$ since inequalities (17) hold. This complete the equivalence proof of the regions $\mathcal{R}_{n o R_{1}}^{o}$ and $\mathcal{R}_{n o R_{1}}^{i}$.

The proof that $\mathcal{R}_{n o R_{1}}^{o}$ is an upper bound, i.e. $\mathcal{C}_{n o R_{1}} \subseteq$ $\mathcal{R}_{n o R_{1}}^{o}$, is similar to the converse part given in Section IV.B. Thus we will give it here shortly:

$$
\begin{aligned}
& n\left(R_{0}-\epsilon_{2, n}\right) \leq I\left(M_{0} ; Y_{2}^{n}\right) \\
& =\sum_{i=1}^{n} I\left(M_{0} ; Y_{2, i} \mid Y_{2, i+1}^{n}\right) \\
& \leq \sum_{i=1}^{n} I\left(M_{0} Y_{2, i+1}^{n} Y_{1}^{i-1} ; Y_{2, i}\right) \\
& n\left(R_{0}+R_{0}^{\prime}-\epsilon_{2, n}^{\prime}\right) \leq I\left(M_{0} M_{0}^{\prime} ; Y_{2}^{n}, \phi\left(Y_{1}^{n}\right)\right) \\
& =I\left(M_{0} M_{0}^{\prime} ; Y_{2}^{n}\right)+I\left(M_{0} M_{0}^{\prime} ; \phi\left(Y_{1}^{n}\right) \mid Y_{2}^{n}\right) \\
& \leq \sum_{i=1}^{n} I\left(M_{0} M_{0}^{\prime} ; Y_{2, i} \mid Y_{2, i+1}^{n}\right)+H\left(\phi\left(Y_{1}^{n}\right)\right) \\
& \leq \sum_{i=1}^{n} I\left(M_{0} M_{0}^{\prime} X_{i} Y_{2, i+1}^{n} ; Y_{2, i}\right)+n C_{1} \\
& =\sum_{i=1}^{n} I\left(X_{i} ; Y_{2, i}\right)+n C_{1} \\
& n\left(R_{0}+R_{0}^{\prime}-\epsilon_{1, n}\right) \leq I\left(M_{0} M_{0}^{\prime} ; Y_{1}^{n}\right) \\
& =\sum_{i=1}^{n} I\left(M_{0} M_{0}^{\prime} ; Y_{1, i} \mid Y_{1}^{i-1}\right) \\
& \leq \sum_{i=1}^{n} I\left(X_{i} M_{0} M_{0}^{\prime} Y_{1}^{i-1} ; Y_{1, i}\right) \\
& =\sum_{i=1}^{n} I\left(X_{i} ; Y_{1, i}\right)
\end{aligned}
$$

$$
\begin{aligned}
n & \left(R_{0}+R_{0}^{\prime}-\epsilon_{2, n}^{\prime}-\epsilon_{1, n}\right) \\
\leq & I\left(M_{0} ; Y_{2}^{n}\right)+I\left(M_{0}^{\prime} ; Y_{1}^{n}\right) \\
\leq & I\left(M_{0} ; Y_{2}^{n}\right)+I\left(M_{0}^{\prime} ; Y_{1}^{n} \mid M_{0}\right) \\
& \sum_{i=1}^{n} I\left(M_{0} ; Y_{2, i} \mid Y_{2, i+1}^{n}\right)+\sum_{i=1}^{n} I\left(M_{0}^{\prime} ; Y_{1, i} \mid M_{0} Y_{1}^{i-1}\right) \\
\leq & \sum_{i=1}^{n} I\left(M_{0} Y_{2, i+1}^{n} ; Y_{2, i}\right)+ \\
+ & \sum_{i=1}^{n} I\left(M_{0}^{\prime} Y_{2, i+1}^{n} ; Y_{1, i} \mid M_{0} Y_{1}^{i-1}\right) \\
= & \sum_{i=1}^{n} I\left(M_{0} Y_{2, i+1}^{n} ; Y_{2, i}\right)+\sum_{i=1}^{n} I\left(Y_{2, i+1}^{n} ; Y_{1, i} \mid M_{0} Y_{1}^{i-1}\right)+ \\
+ & \sum_{i=1}^{n} I\left(M_{0}^{\prime} ; Y_{1, i} \mid M_{0} Y_{1}^{i-1} Y_{2, i+1}^{n}\right) \\
& \stackrel{(a)}{=} \sum_{i=1}^{n} I\left(M_{0} Y_{2, i+1}^{n} ; Y_{2, i}\right)+\sum_{i=1}^{n} I\left(Y_{1}^{i-1} ; Y_{2, i} \mid M_{0} Y_{2, i+1}^{n}\right)+ \\
+ & \sum_{i=1}^{n} I\left(X_{i} M_{0}^{\prime} ; Y_{1, i} \mid M_{0} Y_{1}^{i-1} Y_{2, i+1}^{n}\right) \\
& =\sum_{i=1}^{n} I\left(M_{0} Y_{2, i+1}^{n} Y_{1}^{i-1} ; Y_{2, i}\right)+ \\
+ & \sum_{i=1}^{n} I\left(X_{i} ; Y_{1, i} \mid M_{0} Y_{1}^{i-1} Y_{2, i+1}^{n}\right)
\end{aligned}
$$

where $(a)$ is due to Csiszar sum identity and the fact that $X_{i}$ is a deterministic function of the messages. Finally define $U_{i}=\left(M_{0} Y_{2, i+1}^{n} Y_{1}^{i-1}\right)$ in (19) and then a time-sharing random variable uniformly distributed $Q \sim$ Uniform $[1: n]$ independent of all R.V, and taking the limit $n \rightarrow \infty$ to complete the converse proof.

\section{E. Proof for the $A W G N B C$}

The proof follows by the same arguments of the capacity proof for the AWGN BC. Observe that this channel is stochastically degraded, i.e. there exist $Y_{1}^{\prime}, Y_{2}^{\prime}$ such that $P_{Y_{1} Y_{2} \mid X}=P_{Y_{1}^{\prime} Y_{2}^{\prime} \mid X}$ where

$$
\begin{aligned}
Y_{1}^{\prime}=X+Z_{1}^{\prime} & Z_{1}^{\prime} \sim \mathcal{N}\left(0, N_{1}\right) \\
Y_{2}^{\prime}=Y_{1}^{\prime}+\tilde{Z}_{2} & \tilde{Z}_{2} \sim \mathcal{N}\left(0, N_{2}-N_{1}\right)
\end{aligned}
$$

There are five inequalities in (1), but as the channel is stochastically degraded we have $I\left(U ; Y_{2}\right) \leq I\left(U ; Y_{1}\right)$ and (1d) which makes inequality (1e) redundant. Denote by $h(\cdot)$ the differential entropy of a continuous R.V, and define the Gaussian entropy function and capacity by:

$$
\mathcal{H}(x)=\frac{1}{2} \log (2 \pi e x) \quad \mathcal{C}(x)=\frac{1}{2} \log (1+x)
$$

We bound $h\left(Y_{2} \mid U\right)$ :

$$
\mathcal{H}\left(N_{2}\right)=h\left(Z_{2}\right) \leq h\left(Y_{2} \mid U\right) \leq h\left(Y_{2}\right) \leq \mathcal{H}\left(P+N_{2}\right)
$$

so there exist $\bar{\alpha}_{0} \in[0,1]$ such that

$$
h\left(Y_{2} \mid U\right)=\mathcal{H}\left(\bar{\alpha}_{0} P+N_{2}\right)
$$


where we use $\bar{\alpha}_{0}=1-\alpha_{0}$ for convenience of notation. We can now bound $R_{0}$ :

$$
\begin{aligned}
R_{0} & \leq I\left(U ; Y_{2}\right)=h\left(Y_{2}\right)-h\left(Y_{2} \mid U\right) \\
& \leq \mathcal{H}\left(P+N_{2}\right)-\mathcal{H}\left(\bar{\alpha}_{0} P+N_{2}\right)=\mathcal{C}\left(\frac{\alpha_{0} P}{N_{2}+\bar{\alpha}_{0} P}\right)
\end{aligned}
$$

In order to prove inequality $3 \mathrm{~d}$, we use the scalar EPI:

$$
\begin{aligned}
h\left(Y_{2} \mid U\right) & =h\left(Y_{2}^{\prime} \mid U\right)=h\left(Y_{1}^{\prime}+\tilde{Z}_{2} \mid U\right) \\
& \geq \frac{1}{2} \log \left(2^{2 h\left(Y_{1}^{\prime} \mid U\right)}+2^{2 h\left(\tilde{Z}_{2} \mid U\right)}\right) \\
& =\frac{1}{2} \log \left(2^{2 h\left(Y_{1}^{\prime} \mid U\right)}+2^{2 h\left(\tilde{Z}_{2}\right)}\right) \\
& =\frac{1}{2} \log \left(2^{2 h\left(Y_{1}^{\prime} \mid U\right)}+2 \pi e\left[N_{2}-N_{1}\right]\right)
\end{aligned}
$$

From 20 and 21 we have $h\left(Y_{1}^{\prime} \mid U\right) \leq \mathcal{H}\left(\bar{\alpha}_{0} P+N_{1}\right)$. Thus we can bound $R_{0}^{\prime}+R_{1}$ :

$$
\begin{aligned}
R_{0}^{\prime}+R_{1} & \leq I\left(X ; Y_{1} \mid U\right)=h\left(Y_{1} \mid U\right)-h\left(Y_{1} \mid X\right) \\
& =h\left(Y_{1}^{\prime} \mid U\right)-h\left(Z_{1}\right) \\
& \leq \mathcal{H}\left(\bar{\alpha}_{0} P+N_{1}\right)-\mathcal{H}\left(N_{1}\right)=\mathcal{C}\left(\frac{\bar{\alpha}_{0} P}{N_{1}}\right)
\end{aligned}
$$

Similarly we bound $h\left(Y_{2} \mid U V\right)$ :

$\mathcal{H}\left(N_{2}\right)=h\left(Z_{2}\right) \leq h\left(Y_{2} \mid U V\right) \leq h\left(Y_{2} \mid U\right)=\mathcal{H}\left(\bar{\alpha}_{0} P+N_{2}\right)$

so there exist $\alpha_{1} \in\left[0, \bar{\alpha}_{0}\right]$ such that

$$
h\left(Y_{2} \mid U V\right)=\mathcal{H}\left(\alpha_{1} P+N_{2}\right)
$$

We proceed by bounding the $R_{0}^{\prime}$ :

$$
\begin{aligned}
R_{0}^{\prime} & \leq I\left(V ; Y_{2} \mid U\right)+C_{1}=h\left(Y_{2} \mid U\right)-h\left(Y_{2} \mid U V\right)+C_{1} \\
& =\mathcal{H}\left(\bar{\alpha}_{0} P+N_{2}\right)-\mathcal{H}\left(\alpha_{1} P+N_{2}\right)+C_{1} \\
& =\mathcal{C}\left(\frac{\left[\bar{\alpha}_{0}-\alpha_{1}\right] P}{N_{2}+\alpha_{1} P}\right)+C_{1} \\
& \triangleq \mathcal{C}\left(\frac{\alpha_{0}^{\prime} P}{N_{2}+\alpha_{1} P}\right)+C_{1}
\end{aligned}
$$

where we define $\alpha_{0}^{\prime} \triangleq\left[\bar{\alpha}_{0}-\alpha_{1}\right]$, thus $\alpha_{0}+\alpha_{0}^{\prime}+\alpha_{1}=1$. To prove inequality $3 \mathrm{c}$, we use again the scalar EPI:

$$
\begin{aligned}
h\left(Y_{2} \mid U V\right) & =h\left(Y_{2}^{\prime} \mid U V\right)=h\left(Y_{1}^{\prime}+\tilde{Z}_{2} \mid U V\right) \\
& \geq \frac{1}{2} \log \left(2^{2 h\left(Y_{1}^{\prime} \mid U V\right)}+2^{2 h\left(\tilde{Z}_{2} \mid U V\right)}\right) \\
& =\frac{1}{2} \log \left(2^{2 h\left(Y_{1}^{\prime} \mid U V\right)}+2^{2 h\left(\tilde{Z}_{2}\right)}\right) \\
& =\frac{1}{2} \log \left(2^{2 h\left(Y_{1}^{\prime} \mid U V\right)}+2 \pi e\left[N_{2}-N_{1}\right]\right)
\end{aligned}
$$

From 22 and 23 we have $h\left(Y_{1}^{\prime} \mid U V\right) \leq \mathcal{H}\left(\alpha_{1} P+N_{1}\right)$. Thus we can bound $R_{1}$ :

$$
\begin{aligned}
R_{1} & \leq I\left(X ; Y_{1} \mid U V\right)=h\left(Y_{1} \mid U V\right)-h\left(Y_{1} \mid X\right) \\
& =h\left(Y_{1}^{\prime} \mid U V\right)-h\left(Z_{1}\right) \\
& \leq \mathcal{H}\left(\alpha_{1} P+N_{1}\right)-\mathcal{H}\left(N_{1}\right)=\mathcal{C}\left(\frac{\alpha_{1} P}{N_{1}}\right)
\end{aligned}
$$

For the achievability we define three independent zeromean Gaussian random variables $U, V, W$ with variances $\alpha_{0} P, \alpha_{0}^{\prime} P, \alpha_{1} P$ respectively. Define $X=U+V+W$ and plug in inequalities (1) to get inequalities (3).

Remark. The capacity region is represented as a union of regions defined by three parameters $\alpha_{0}, \alpha_{0}^{\prime}, \alpha_{1}$, although each region is determined by only two of them as $\alpha_{0}+\alpha_{0}^{\prime}+\alpha_{1}=1$. The reason becomes clear if one observes that each of those $\alpha$ 's, represents the power $\alpha P$ dedicated to each message. For example, inequality (3a) stands for the decoding procedure of decoder 2 , when $m_{0}$ is the first (and sometimes the only) message to decode. Thus, treating the power of the messages $m_{0}^{\prime}, m_{1}$ as additional noise, set the SNR to be $\frac{\alpha_{0} P}{N_{2}+\left[\alpha_{0}^{\prime}+\alpha_{1}\right] P}$.

\section{REFERENCES}

[1] R. Dabora and S. Servetto, "Broadcast Channels with Cooperating Receivers : A Downlink for the Sensor Reachback Problem," in Proc. IEEE Int. Symp. Information Theory, Chicago, IL, June 27-July 2, 2004, p. 176.

[2] R. Dabora and S. D. Servetto, "Broadcast Channels With Cooperating Decoders," IEEE Transactions on Information Theory, vol. 52, no. 12, pp. 5438-5454, 2006.

[3] Y. Liang and V. V. Veeravalli, "Cooperative Relay Broadcast Channels," IEEE Transactions on Information Theory, vol. 53, no. 3, pp. 900-928, 2007.

[4] Yingbin Liang and V. Veeravalli, "The Impact of Relaying on the Capacity of Broadcast Channels," in Proc. IEEE Int. Symp. Information Theory, Chicago, IL, June 27-July 2, 2004, p. 403. IEEE, 2004, p. 403.

[5] Y. Steinberg, "Instances of the Relay-Broadcast Channel and Cooperation Strategies," in IEEE International Symposium on Information Theory Proceedings, Hong Kong, 2015.

[6] Y. Steinberg, "Channels with Cooperation Links that May Be Absent," in Proc. IEEE International Symposium on Information Theory - Honolulu, HI, June 29-July 4,, 2014.

[7] W. Huleihel and Y. Steinberg, "Channels with Cooperation Links that May Be Absent," Submitted paper, 2016.

[8] J. Körner and K. Marton, "General Broadcast Channels with Degraded Message Sets," IEEE Transactions on Information Theory, 1977.

[9] R. T. Rockafellar, Convex Analysis, 1970.

[10] M. Henk, J. Richter-Gebert, and G. M. Ziegler, Handbook of Discrete and Computational Geometry, Ch.15 Basic properties of convex polytopes, 1997.

[11] A. El Gamal and Y.-H. Kim, Network Information Theory. Cambridge: Cambridge University Press, 2011. 